MATEC Web of Conferences 32, 06002 (2015)

DOI: $10.1051 /$ matecconf/20153206002

O) Owned by the authors, published by EDP Sciences, 2015

\title{
Microrobotic Station for Nano-Optical Component Assembly
}

\author{
Bilal Komati ${ }^{1, a}$, Cédric Clévy ${ }^{1}$, Nadège Courjal ${ }^{2}$, Gwenn Ulliac $^{2}$ and Philippe Lutz ${ }^{1}$ \\ ${ }^{1}$ FEMTO-ST Institute AS2M department, Univ.Bourgogne Franche-Comté, Univ. de Franche-Comté/CNRS/ENSMM, 24 rue Savary, F- \\ 25000 Besançon, France. \\ ${ }^{2}$ FEMTO-ST Institute, Optics department, Univ.Bourgogne Franche-Comté, Univ. de Franche-Comté/CNRS/ENSMM, 24 rue Savary, F- \\ 25000 Besançon, France.
}

\begin{abstract}
Compact photonic structures have strategic importance in several fields. In order to fabricate more complex structures with optimized optical function, robotic nano-assembly is a promising solution because it enables to integrate several types of materials from different fabrication processes into the same structure. This paper presents a microrobotic station which is designed for the assembly of nano-optical components. The robotic station has 8-DOF for positioning and 4-DOF microgripper with integrated force sensors to perform dexterous and accurate manipulation of components by considering both position and contact force to achieve precise alignment and parallelism of structures.
\end{abstract}

\section{Introduction}

The development of compact photonic structures presents strategic issues in many fields: biomedical, astrophysics, multimedia, defence, etc. These structures are very complex to fabricate (limits in terms of precision machining, monolithic, diversity of shapes obtainable) but also to study (coupling difficulties, local analysis of the optical behaviour).

Several works have studied the importance of thin metal plates periodically structured by subwavelength holes which present special or enhanced transmission of resonance excitation (plasmonic modes or guided wave sub-length cavities) [1,2]. For optical frequencies (visible/near infrared), the main limiting factor of such structures remains the absorption of metal that dramatically reduces the light transmission. In addition, it should be noted that their multilayer character adds new experimental constraints (such as alignment and parallelism) of their nanoscale dimensions $[3,4]$.

Robotic nano-assembly presents an interesting approach to develop and fabricate more complex photonic structures by assembling several nano-structures issued from different fabrication process. It enables to ensure precise alignment and parallelism of the structures to maximize the optical function. In literature several works have studied robotic nano-assembly including the use of scanning electron microscopes (SPM), atomic force microscopes (AFM), scanning electron microscope (SEM) and nano-tweezers [5]. The main issue is the size of the component to manipulate but also the predominance of surface and contact forces at this scale relative to the volume forces such as weight of the nanostructures. Integrating force sensors to measure these

\footnotetext{
a Corresponding author: bilal.komati@femto-st.fr
}

forces is an interesting approach to master the scaling effect, increase the dexterity for the assembly of small components, avoid breaking or damaging components that have very high dimensions ratio and control any contact between different photonic components.

Based on state of the art and previous works which were conducted for microassembly of hybrid MOEMS [6-8], we propose in this paper a new microassembly station for nano-optical component assembly. For this reason, a robotic station made of precise positioning and rotation stages and a new two-sensing-fingersmicrogripper are used to perform dexterous microassembly. The objective of the use of the proposed robotic station is to offer a new range of threedimensional photonic structures and heterogeneous integrating different functionalities by nano-robotic assembly.

The paper is organized as follows. Section 2 introduces some potential applications. The robotic station including a two-sensing fingers microgripper are presented in section 3. Experimental results are then presented in section 4 . Section 5 concludes the paper.

\section{Nano-optical component assembly}

The fabrication of nano-optical components have been widely studied and investigated due to its importance for several domains. Previous works have been interested in the fabrication of several types and forms of photonic structures (shown in Figure 1) using cutting and Focused Ion Beam (FIB). Recently, a photonic cavity on a $\mathrm{LiNbO}_{3}$ membrane leaded to a transmission peak, whose spectral position is extremely sensitive to the temperature for an active length of only 
$13 \mu \mathrm{m}$. All these preliminary results on lithium niobate are very attractive, but it is possible to go even further towards integrated structures. In assembling these nanoguides with metallic-dielectric and polarization control, a decisive step can be taken towards integrated miniaturized electro-optical components with total space less than $1 \mathrm{~mm}^{3}$ and low power consumption.

Fabricating hybrid nano-optical structures by robotic micro-assembly approach requires considering several locks:

- The small size of the components to be manipulated (tens of $\mu \mathrm{m}$ sideway and hundreds nanometers thickness)

- The high aspect ratio of components which induces fragility

- Assembly requires to control all 6 Degrees of Freedom of the manipulated component relative to a reference with high accuracy (better than $1 \mu \mathrm{m}$ as a first step)

- Assembly requires to minimize air gaps between components and thus to maximize contact between assembled components (i.e. controlling contact forces)

(a)

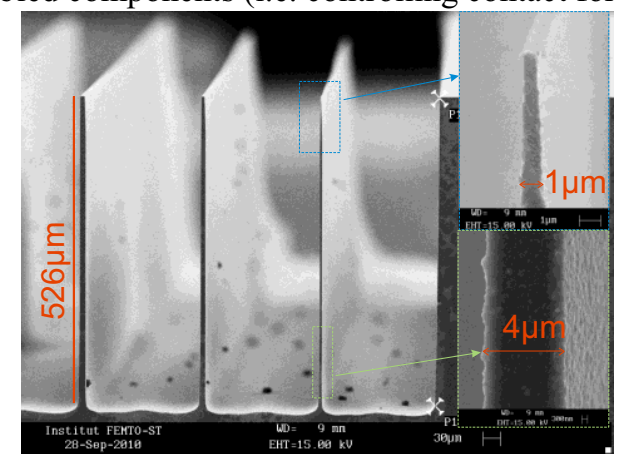

(b)

(c)

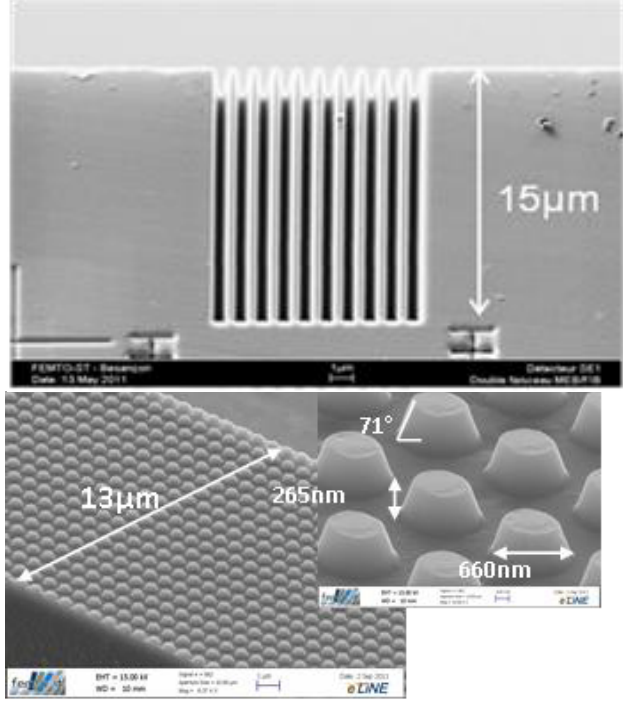

Figure 1. Examples of photonic structures: (a) Ridges obtained by saw dicing and polishing of the bulk material (b) 3D photonic cristal onto a membrane (c) photonic component with surface structured by small bumps.

\section{Microrobotic station for the assembly}

\subsection{Presentation of the robotic station}

The robotic microassembly station proposed in this paper is shown in Figure 2. The experimental setup is composed of large range motorized stages, fine positioning stages, rotation stages and a microgripper with integrated force sensors. The large range motorized stages are M-112.1DG from PI (Physik Instrumente) to perform large range $\mathrm{X}, \mathrm{Y}$ and $\mathrm{Z}$ displacement up to 15 $\mathrm{mm}$. The fine positioning stage is a P-611.3 NanoCube with $100 \mu \mathrm{m}$ range and $1 \mathrm{~nm}$ in resolution with internal position sensors. Two rotation stages from SmarAct SR3610-S with $1.1 \mu^{\circ}$ in resolution are used to adjust the alignment and the parallelism in the robotic station. The positioning and rotation stages are sensorized and closed loop controlled for higher position control accuracy.

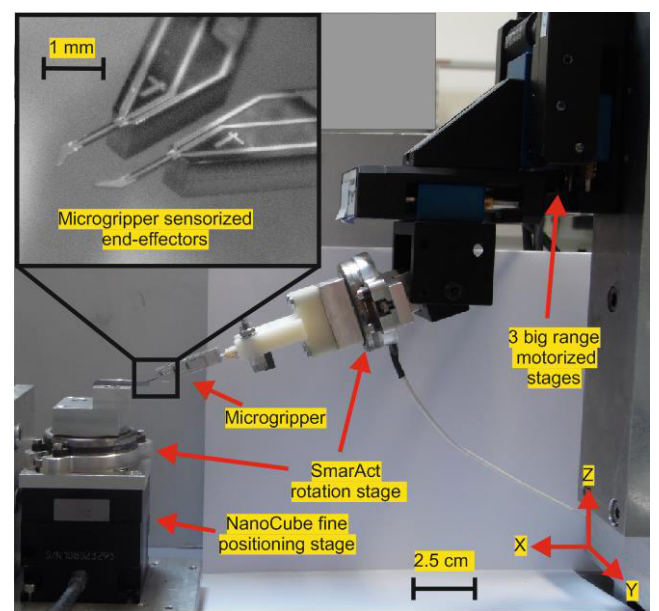

Figure 2. Robotic microassembly station proposed for the nano-assembly composed of 8-DOF positioning and rotation stages, 4-DOF microgripper with sensorized end-effectors.

\subsection{Microgripper with sensorized end-effectors}

In the last decade, several researches have been interested by the development of force sensors for micro and nano scale applications. In literature, the most used solution is to use a microgripper with an active finger and a force sensing finger. However, in previous works [9], the use of two-sensing-fingers microgripper provides an estimation of the lateral contact force between the manipulated micropart and the microassembly substrate in addition to the gripping forces.

In the microgripper design, two main points should be considered: the dimensions of the end-effectors and the force sensing and displacement specifications. For the first point, the force sensor has been designed to manipulate small components such as $7 \times 7 \mu \mathrm{m}$ parts with great dexterity. For this reason, the thickness of the force sensor is $10 \mu \mathrm{m}$. For the microgripper specifications, the solution of using two active fingers provides more dexterity to achieve more complex tasks. Thus, the microgripper used is composed of two active fingers with integrated force sensors. Indeed, a piezoresistive force sensor [10] is integrated into each finger of a piezoelectric actuator [11] to realize a dexterous microgripper. An image of the one force sensor is shown in Figure 3 where the dimensions of the force sensor are 
shown. Particularly, its dimensions are $1 \mathrm{~mm}$ of length, $100 \mu \mathrm{m}$ of width and $10 \mu \mathrm{m}$ of thickness.

The proposed TSFM performances have been identified experimentally for each of the fingers actuator and force sensor. The performances of the TSFM are summarized in Table 1. The performances of the TSFM enable its use for precise nano-assembly while measuring nanonewton forces.

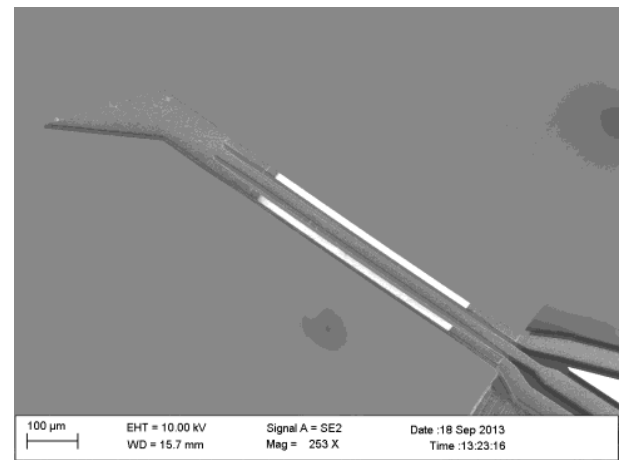

Figure 3. Force sensor integrated inside the microgripper $[10]$.

Table 1. Performances of each finger of the TSFM.

\begin{tabular}{|c|c|c|}
\hline \multirow{2}{*}{ Displacement } & Resolution & $1 \mathrm{~nm}$ \\
\cline { 2 - 3 } & Range & $100 \mu \mathrm{m}$ \\
\hline \multirow{2}{*}{ Force sensing } & Resolution & $60 \mathrm{nN}$ \\
\cline { 2 - 3 } & Range & $2 \mathrm{mN}$ \\
\hline
\end{tabular}

\section{Experimental results}

In this section, some preliminary experimental investigations are done to provide a proof of concept of the possibility of performing dexterous nano-assembly with the presented robotic station. A handling test, for a component with $300 \mu \mathrm{m}$ of length, $50 \mu \mathrm{m}$ of width and $50 \mu \mathrm{m}$ of thickness, is shown in Figure 4-(a) where the forces from both sides of the system are measured precisely and the contact transitions are detected. Figure 4-(b) shows a detection of an undesired contact between the manipulated component and the substrate while the assembly. The undesired contact can be detected using the two force measurements and the contact force can be estimated as the difference $\Delta \mathrm{f}$ (Figure 4-(b)).

(a)

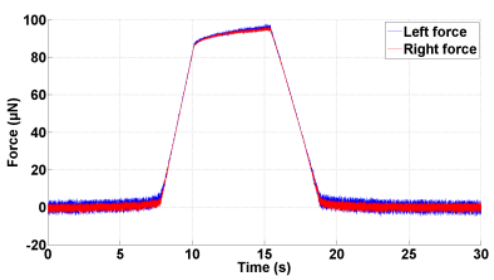

(b)

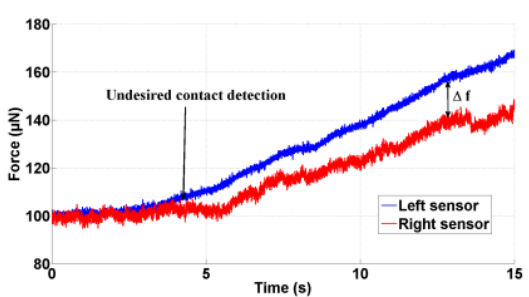

Figure 4. (a) Handling test using the microgripper (b) undesired contact detection.

\section{Conclusion}

The motivation of this paper is the need of high performant photonic structures for many application fields. Robotic nano-assembly has been presented as a method to fabricate complex photonic structures with optimized optical functionalities. Indeed, it enables to integrate several types of materials from different fabrication processes into the same structure. This paper has proposed a new robotic structure to realize this nanoassembly. The robotic station has 8-DOF for positioning and 4-DOF microgripper with integrated force sensors to perform dexterous manipulation of microparts by detecting the contact and by providing precise alignment and parallelism of structures. An experimental proof of concept for the nano-assembly has been validated by testing the results of manipulation and assembly of components where there dimensions are bigger than photonic structures. The gripping forces have been measured and the contact has been detected precisely enabling to perform dexterous nano-assembly.

Future works include the test of this robotic station for the assembly of photonic structures while characterising the precision of the robotic station and the optical results.

\section{Acknolwledgments}

These works have been funded by the Franche-Comté region through the project nano-ROBOPTIC, supported by the Labex ACTION project (contract "ANR-11-LBX0001-01") and by the French RENATECH network through its FEMTO-ST technological facility.

\section{References}

1. T.W. Ebbesen, H.J. Lezec, H.F. Ghaemi, T. Thio, P. A. Wolff, Nature, 391, 667-669 (1998)

2. F.I. Baida, D. Van Labeke, Opt. Comm., 269, 17-22 (2002)

3. Z. Chen, C. Wang, Y. Lou, B. Cao, X. Li, Opt. Comm., 297, (2013).

4. Z.H. Zhu, C.C. Guo, K. Liu, W.M. Ye, X.D. Yuan, B. Yang, T. Ma, Opt. Lett., 37, (2012).

5. Zuobin Wang, S. Fatikow, Shizhong Su, Ming Yang, International Conference on Mechatronics and Automation, 422-427, (2007). ICMA 2007.

6. S. Bargiel, K. Rabenorosoa, C. Clévy, C. Gorecki, P. Lutz, J. Micromech. Microeng., 20 (2010).

7. C. Clévy, I. Lungu, K. Rabenorosoa, P. Lutz, Assembly Automation, 34, 68-77, (2014).

8. B. Komati, K. Rabenorosoa, C. Clévy, P. Lutz, IEEE Transactions on Automation Science and Engineering (T-ASE), 10, 515-524, (2013)

9. K. Rabenorosoa, C. Clévy, Q. Chen, P. Lutz, IEEE/ASME Transactions on Mechatronics (TMech), 811-821, 17 (2012).

10. B. Komati, J. Agnus, C. Clévy, P. Lutz, J. Micromech. Microeng., 24, 035018, (2014)

11. P. de lit, J. Agnus, C. Clévy, N. Chaillet, Assembly and automation, 24, 33-42 (2004). 